\section{Multimorbidity and GP burnout}

This important paper by Pederson and colleagues provides evidence of a relationship between GP burnout and patient multimorbidity in practices in Denmark. ${ }^{1}$ This relationship was significant in unadjusted analysis, but disappeared when adjusted for patient age and sex, leading the authors to conclude that the burden of multimorbidity of older patients added to 'actual work pressure'. This conclusion, if correct, means that practices with older patients have greater work pressure, and GPs in such practices will be at greater risk of burnout.

Before such a conclusion can be reached, there are critical points to consider. It is established that multimorbidity is more common and occurs at an earlier age in deprived areas in high-income countries, ${ }^{2,3}$ including Denmark. ${ }^{4} \mathrm{~A}$ previous study by the same group showed higher burnout in GPs working in deprived areas. ${ }^{5}$ These studies seem incompatible with the current findings.

The answer is likely to lie in how multimorbidity was defined and measured in this study. Multimorbidity was operationalised as two or more physical chronic conditions, as recorded in secondary care data. Such a definition will have two effects: to underestimate the true prevalence of multimorbidity in primary care and to exclude the contribution that mental health conditions make to multimorbidity lusually defined as two or more mental and/or physical conditions).

Our conjecture is that the management of mental-physical multimorbidity is more challenging than physical multimorbidity, especially if physical conditions are concordant. In our previous work, the common physical conditions in those with multimorbidity aged $>75$ years were all concordant: hypertension, coronary heart disease, chronic kidney disease, diabetes, and stroke. ${ }^{6}$ In deprived areas, where patients are younger, the common physical morbidities are discordant (chronic pain, asthma, hypertension). ${ }^{6}$ The combination of discordant physical conditions, a range of mental illnesses (for example, addiction, depression), ${ }^{6}$ and the social problems that patients present in deprived areas ${ }^{7}$ demands a holistic generalist response and has been shown to lead to increased GP stress. ${ }^{7}$ It is essential that this is fully taken into account before reaching conclusions around the relationship of GP burnout to multimorbidity.

Stewart W Mercer,

Professor of Primary Care and

Multimorbidity, Usher Institute, University

of Edinburgh, Edinburgh.

Email: stewart.mercerlaed.ac.uk

Graham CM Watt,

Emeritus Professor of General Practice, University of Glasgow, Glasgow.

Johanna Reilly,

Clinical Academic Fellow in General

Practice, University of Edinburgh,

Edinburgh

Anne Mullin,

GP and Chair of the GPS at the Deep End Steering Group, Govan Health Centre, Glasgow.

[On behalf of the Deep End Steering Group.]

\section{REFERENCES}

1. Pedersen AF, Nørøxe KB, Vedsted P. Influence of patient multimorbidity on GP burnout: a survey and register-based study in Danish general practice. Br J Gen Pract 2020; DOI: https://doi. org/10.3399/bjgp20X707837.

2. Barnett $\mathrm{K}$, Mercer SW, Norbury M, et al. Epidemiology of multimorbidity and implications for health care, research, and medical education: a cross-sectional study. Lancet 2012; 380(9836): $37-43$

3. Violan C, Foguet-Boreu Q, Flores-Mateo G, et al. Prevalence, determinants and patterns of multimorbidity in primary care: a systematic review of observational studies. PLOS One 2014; 9(7): e102149.

4. Willadsen T, Siersma V, Nicolaisdóttir D, et al. Multimorbidity and mortality: a 15-year longitudinal registry-based nationwide Danish population study. J Comorb 2018; 8(1): DOI: 10.1177/2235042X18804063.

5. Pedersen AF, Vedsted P. Understanding the inverse care law: a register and survey-based study of patient deprivation and burnout in general practice. Int J Equity Health 2014; 13(1): 121.

6. McLean G, Guthrie B, Mercer SW, Watt GCM. General practice funding underpins the persistence of the inverse care law: cross- sectional study in Scotland. Br J Gen Pract 2015; DOI: https://doi.org/10.3399/bjgp15X687829.

7. Mercer SW, Watt GCM. The inverse care law: clinical primary care encounters in deprived and affluent areas of Scotland. Ann Fam Med 2007. 5(6): 503-550.

DOI: https://doi.org/10.3399/bjgp20X708353

\section{Mental health in diabetes: can't afford to address the service gaps or can't afford not to?}

I must admit that when the BJGP arrived, and I started this article, ${ }^{1}$ I became cross and didn't finish it. The paper version was of course recycled. But, a couple of patients whose issues piqued my curiosity in the first place brought me back to it online. It still irked and then a throwaway comment in the final paragraph seemed to suggest, well all right then

I have no issues at all with the service described here but I think that final comment has things back to front. Yes, complex mental health issues in diabetes are an unmet need. Yes, this deserves to be funded properly. However, what is needed is not a diabetes-focused, or even long-termcondition-focused, mental health service. What is needed and has been for a very long time (l've been a doctor for 34 years, a GP for 27 of those, and I can't remember a time when mental health was adequately provided forl is a comprehensive, flexible mental health service.

If such were available, then I wouldn't have to fight for every single child or young person referral to be accepted and Adverse Childhood Episodes would be better managed. If such were available, then all of my patients with long-term conditions would get the support they need to optimise their management. For far too long the mind-body (false) dichotomy has failed to grasp the interdependence of physical and mental wellbeing. Funding is indeed needed in a sustainable fashion for mental health 